\title{
A Classical Molecular Dynamics Study into the Effect of Si/Al Ratio and Silanol Nest Defects on Water Diffusion in Zeolite HY
}

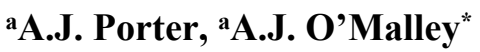

${ }^{a}$ Centre for Sustainable and Circular Technologies, Department of Chemistry, University of Bath, UK, BA2 7AY

*a.o'malley@bath.ac.uk

\section{Supplementary information}

\section{$\underline{\text { SI.1 Forcefield Validation }}$}

We have used a relatively novel forcefield combination to describe this system using a mixture of Morse, Buckingham and Lennard-Jones potentials. Before presenting data on the diffusivity behaviour of water in the system, we now demonstrate the viability of this forcefield combination through comparison of obtained adsorption energies of water to the Brønsted acid and silanol nest sites in the zeolite - with those obtained by experiment and quantum mechanical calculations. A single water molecule was placed within an FAU unit cell which contained either of the sites of interest (a single Brønsted acid site or silanol nest) and the adsorption energy calculated via energy minimisation using the conjugate gradient method in DLPOLY4. The adsorption energies obtained for a single water molecule to a BAS were $-58.8 \mathrm{~kJ}$ and that of a single water molecule to a silanol nest to be $-77 \mathrm{~kJ} \mathrm{~mol}^{-}$ 1. An example of the optimised geometry obtained to the Brønsted acid site is shown in figure S.1 consistent with H-bond lengths and geometries seen both later in this article and the QM studies discussed in this section ${ }^{1,2}$.

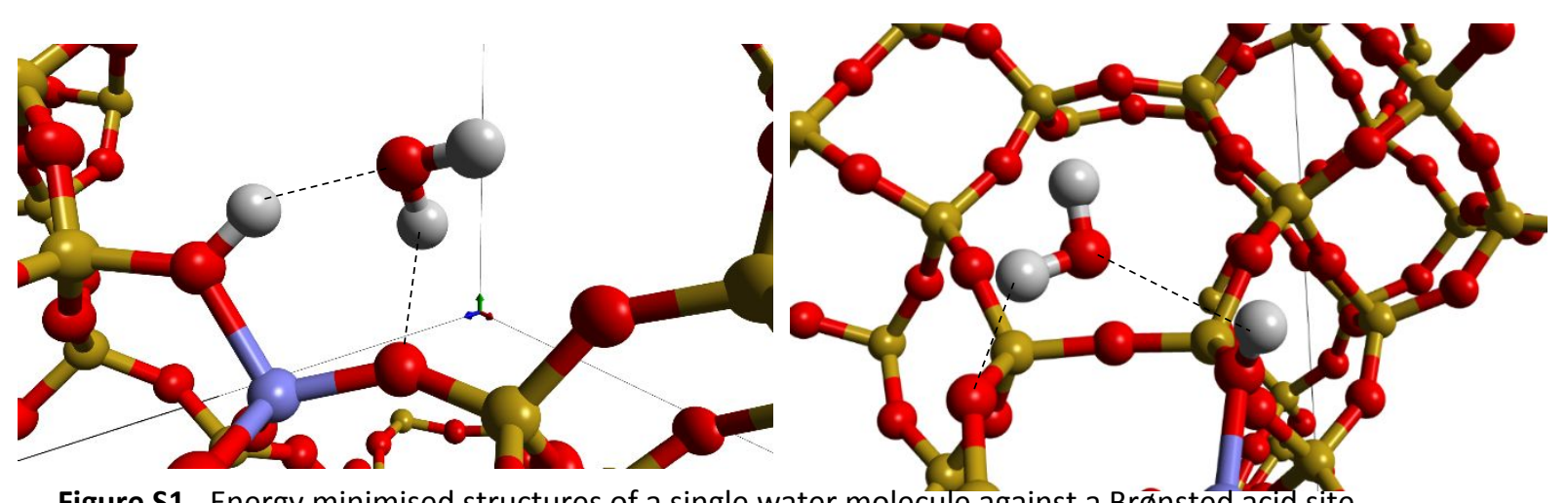

Figure S1. Energy minimised structures of a single water molecule against a Brø̄nsted acid site within a faujasite unit cell. Parts of the framework have been removed for clarity.

We begin with a comparison to experimental values of the heats of adsorption in zeolites, where we note that experimental studies in Brønsted acidic zeolites are very rare. Farrell et al ${ }^{3}$ experimentally 
probed water adsorption in $\mathrm{H}-\mathrm{FAU}$ with a $\mathrm{Si} / \mathrm{Al}$ ratio of 80 and found the isosteric heat of adsorption to be between -34 to $-39 \mathrm{~kJ} \mathrm{~mol}^{-1}$, while we note that their coverages are significantly larger than that simulated here with loadings from $\sim 10$ to $\sim 40 \mathrm{wt} \%$. Eckstein et al. ${ }^{4}$ reported a heat of adsorption for water on a BAS in H-MFI (Across a range of $\mathrm{Si} / \mathrm{Al}=15-110$ ) as $65 \pm 5 \mathrm{~kJ} \mathrm{~mol}^{-1}$ via gas-phase adsorption experiments. While several factors may cause discrepancy such as acid site density and the total coverage of water molecules (which will inevitably be higher than 1 molecule per unit cell as used in our calculation) we consider our adsorption energies to be in reasonable agreement with experimental values as they fall in this range.

In terms of quantum mechanical calculations, it is typically observed that calculated adsorption energies are significantly higher than those obtained experimentally. Though again specific studies of water in H-FAU zeolites are very rare. Examples include Krossner and Sauer ${ }^{2}$ who investigated adsorption of water in H-FAU and calculated the adsorption energy to be $-79.4 \pm 21.6 \mathrm{~kJ} \mathrm{~mol}^{-1}$ via the MP2 method or $-73.0 \pm 14.2 \mathrm{~kJ} \mathrm{~mol}^{-1}$ via a combination of MP2 and Hartree-Fock methods, where our values are in range when the basis set superposition error is taken into account. The majority of quantum mechanical studies of water adsorption on proton containing zeolites probe adsorption in H-ZSM-5, where more recently, Mei and Lercher ${ }^{5}$ probed adsorption in $\mathrm{H}-\mathrm{ZSM}-5(\mathrm{Si} / \mathrm{Al}=23)$ adsorption energy of $-76 \mathrm{~kJ}$ mol- $^{-1}$ at the T4 site. Further studies of water adsorption in H-ZSM-5 include those by Joshi et $\mathrm{al}^{1}$ who extensively reparametrized the ReaxFF forcefield ${ }^{6}$ using DFT calculations to optimise it's use in acidic zeolite systems, finding an adsorption energy of $-64.4 \mathrm{~kJ} \mathrm{~mol}^{-1}$, they also noted an excellent agreement with obtained diffusion coefficients from PFG-NMR experiments ${ }^{7}$ using said forcefield, which gives a similar $E_{a d s}$ to our own, though the differing framework topology and pore size should be taken into account. We therefore consider that our forcefield can be considered reliable for describing the interaction of water with a Brønsted acid site, where our value for the adsorption energy falls within the range of values obtained through a range of experimental measurements and quantum mechanical calculations, including those used to parameterize forcefields for different zeolites which give $\mathrm{D}_{\mathrm{s}}$ values that agree strongly with experimental water diffusivity measurements.

Studies of the heats of adsorption of a water molecule onto a zeolite silanol nest are scarcer. Yazaydin and Thompson ${ }^{8}$ reported heats of adsorption of water to silanol containing MFI type zeolites of c.a. -25 to $-55 \mathrm{~kJ} \mathrm{~mol}^{-1}$ via molecular dynamics simulations, and verified these values through comparison versus a range of experimental gas adsorption results from literature ${ }^{9-13}$ which range from 25.1 to 50.6 $\mathrm{kJ} \mathrm{mol}^{-1}$. Similarly, Ahunbay ${ }^{14}$ used Monte Carlo simulations to probe water adsorption in a similar system of silanol containing MFI type zeolites, providing heats of adsorption from c.a. -30 to $-65 \mathrm{~kJ}$ $\mathrm{mol}^{-1}$. In both cases the authors note significant effects of both the water loading and the concentration of silanol nests, which again differ significantly from our systems. We note that the referenced work does not directly compare the difference in adsorption energy with a BAS. Again, we consider that the 
discrepancies here between our values and those referenced may well be due to the difference in zeolite structure, where the cavity size and local structural relaxation phenomena may play a role. Our values of the adsorption energy however appear to be within a sensible range of those obtained experimentally and we consider our forcefield to be adequate for determining trends in water diffusivity.

\section{$\underline{\text { SI.2 Contact correlation function }}$}

To probe the interactions between the water molecules and the Brønsted acid sites, specifically in terms of residence times, the contact correlation function between $\mathrm{O}_{w}$ and $\mathrm{H}_{\mathrm{b}}$ was also calculated, using equation 2:

$$
C(t)=\frac{1}{N} \sum_{i=1}^{N} \frac{\left\langle p_{i}(t)\right\rangle}{\left\langle n_{i}\left(t_{0}\right) n_{i}\left(t_{0}\right)\right\rangle}
$$

Where $\mathrm{N}$ is the total number of molecules adsorbed at the initial time $t_{0}$, and $p_{i}(t)$ is defined as:

$$
p_{i}(t)=n_{i}\left(t_{0}\right) \prod_{t_{0}}^{t} n_{i}(t)
$$

where $n_{i}(t)$ is a binary function which equals 1 when the two molecules are within the specified contact distance and 0 when they are not. In this work the contact distance was defined as 3.0 A. This can be directly related to the behaviour within the first coordination shell which is seen in later RDFs (i.e. figure 11 whereby the first shell is seen below $3 \AA$ ). An average over several trajectories, beginning at different initial times, was calculated. The contact correlation function may be used to define the residence time, $t_{r}$ of a water molecule at a Brønsted site:

$$
t_{r}=\int_{0}^{\infty} d t C(t)
$$

The residence time is based on the relation time, $\tau=t_{r}$, when the contact correlation decays exponentially i.e. $C(t)=e^{-1 / \tau}$. $t_{r}$ thus corresponds to the time at which $C(t)=e^{-1}=0.3679 .{ }^{52}$ To achieve a single exponential function, and thus one residence time, the contact distance must be sufficiently small to only observe behaviour within one coordination sphere, in this case $3 \AA$. Linear combinations of similar exponential functions may be fit to describe multiple different behaviours over a larger cutoff distance. Once the behaviour within the first coordination sphere has been, described more exponentials may be fit over a larger cutoff distance however this was outside the scope of this work. 

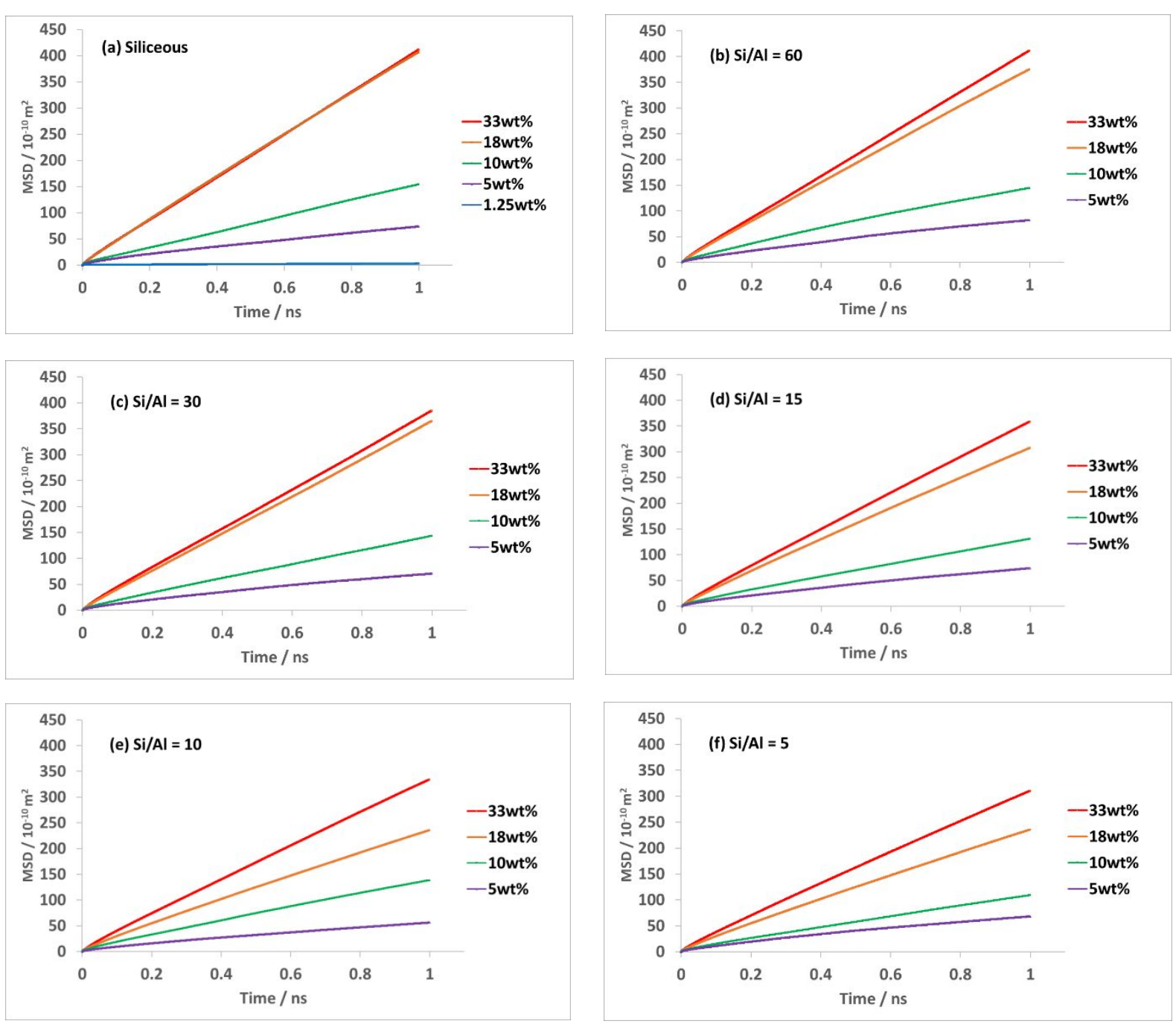

Figure S2. Mean squared displacement plots of $1.25 \mathrm{wt} \%$ (siliceous only), $5 \mathrm{wt} \%, 10 \mathrm{wt} \%, 18 \mathrm{wt} \%$ and $33 \mathrm{wt} \%$ water loading in the fully siliceous and $\mathrm{Si} / \mathrm{Al}=60,30,15,10$ and 5 zeolite $\mathrm{Y}$ systems.

\section{$\underline{\text { SI.3 Water clustering }}$}

The clustering of water molecules may be examined in greater detail by calculation of the local $\mathrm{n}(\mathrm{r})$ of $\mathrm{O}_{\mathrm{w}}-\mathrm{O}_{\mathrm{w}}$ interactions (after integration under the radial distribution function) within the first coordination sphere - this was taken as the minima after the first peak at $\sim 2.75 \AA$ at $3.45 \AA$ - giving the average coordination number of a water molecule to its nearest neighbours. The resulting coordination numbers are shown in figure S3. The water logically shows a greater coordination number as the loading is increased, by factor of $\sim 5-6$ from a loading of $5 \mathrm{wt} \%$ to $33 \mathrm{wt} \%$. The trend in cluster size with water loading can be tentatively considered to correlate with the increase in diffusivity. Whether this correlation is directly proportional is not clear, particularly at higher loadings where between the 18 and $33 \mathrm{wt} \%$ systems, an increase in the cluster size by a factor of 0.65 in the $\mathrm{Si} / \mathrm{Al}=5 \mathrm{system}$ is accompanied by an increase in $\mathrm{D}_{\mathrm{s}}$ of by a factor of 0.35 . Minor fluctuations in the coordination number with the $\mathrm{Si} / \mathrm{Al}$ ratio are observed but no clear trend is present. . At higher loadings ${ }_{2}$ a very slight increase in cluster size may be noted between $\mathrm{Si} / \mathrm{Al}=5$ and 10 but it is difficult to consider this significant. 


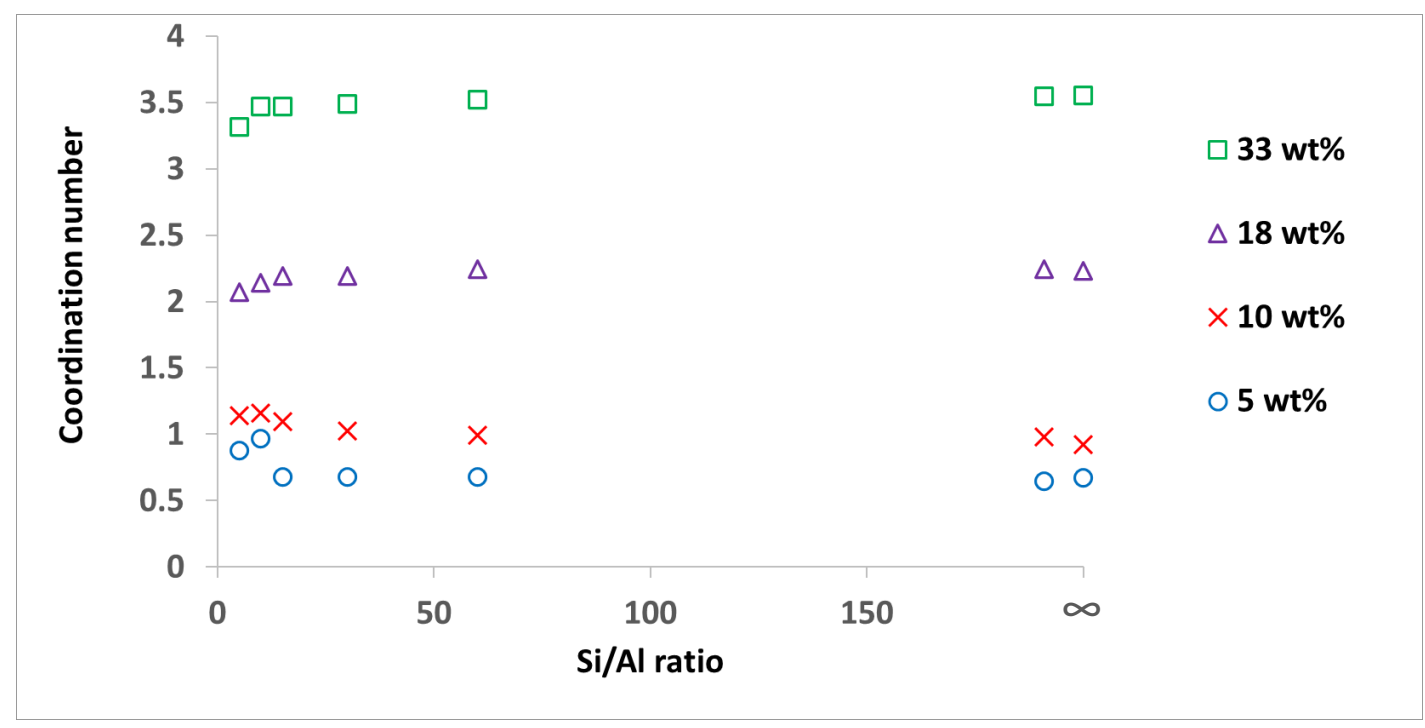

Figure S3. Coordination numbers of water at 5, 10, 18 and 33 wt\% loading in the Si/AI = 5, 10, 15, 30, 60 and 191 zeolite $Y$ systems.
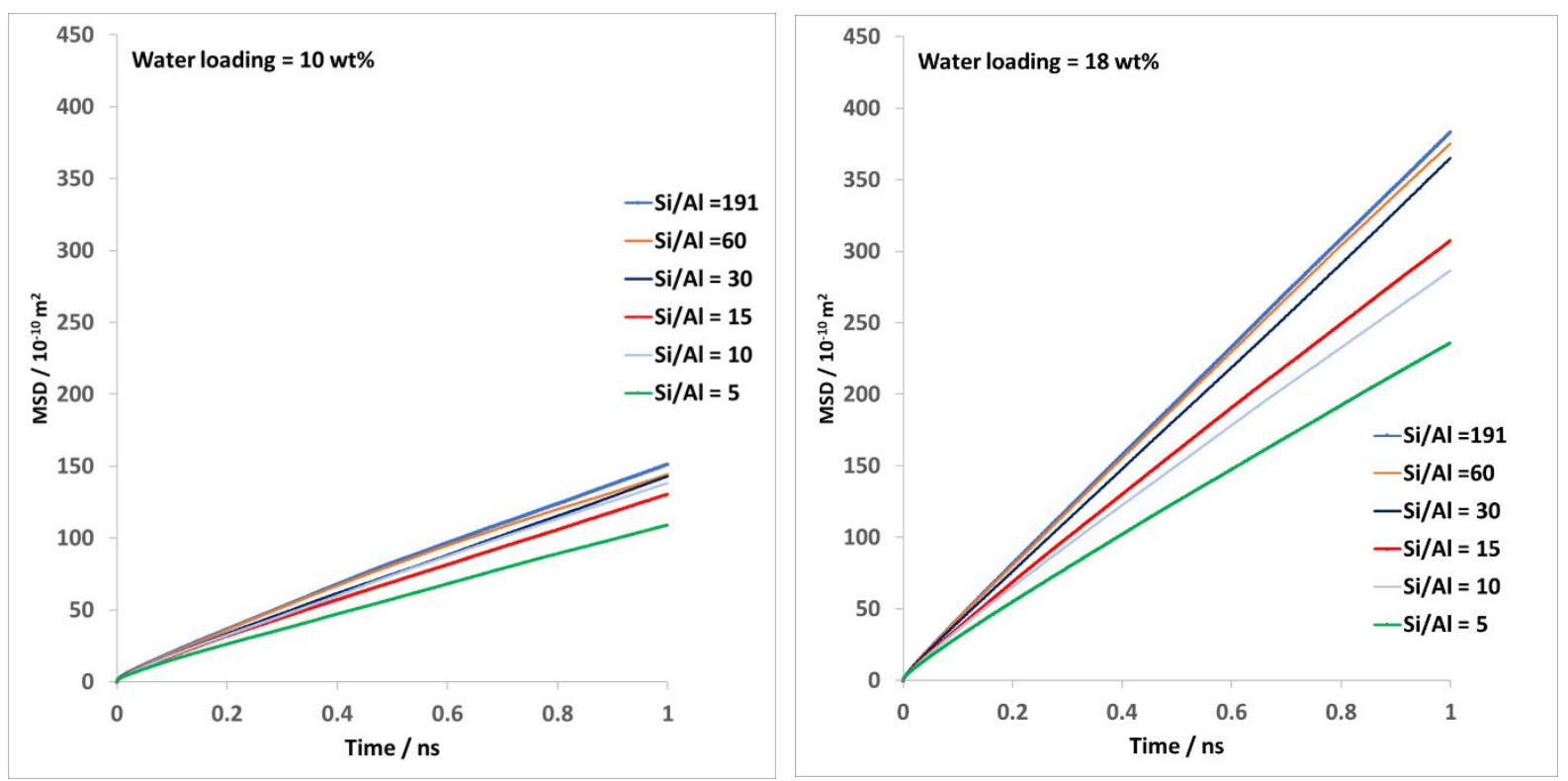

Figure S4. MSD plot of water at 10 and 18 wt\% loadings in the Si/AI = 5, 10, 15, 30, 60 and 191 zeolite $Y$ systems. 


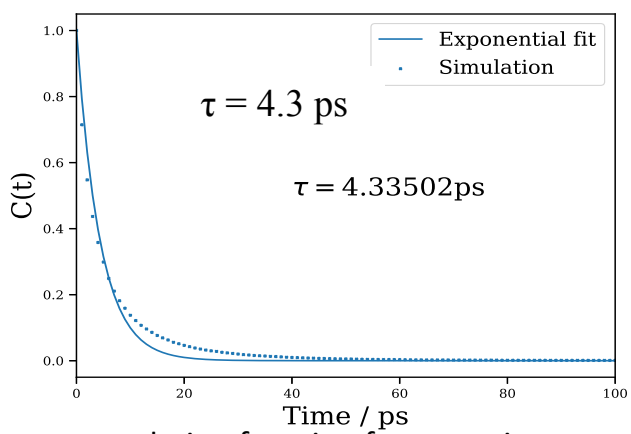

$\tau=2.6 \mathrm{ps}$

Figure S5. Contact correlation function for water in contact with Brønsted acid sites in zeolite HY with Si/Al ratio $=5$ at $5 \mathrm{wt} \%$ (left) and $33 \mathrm{wt} \%$ (right) loading.

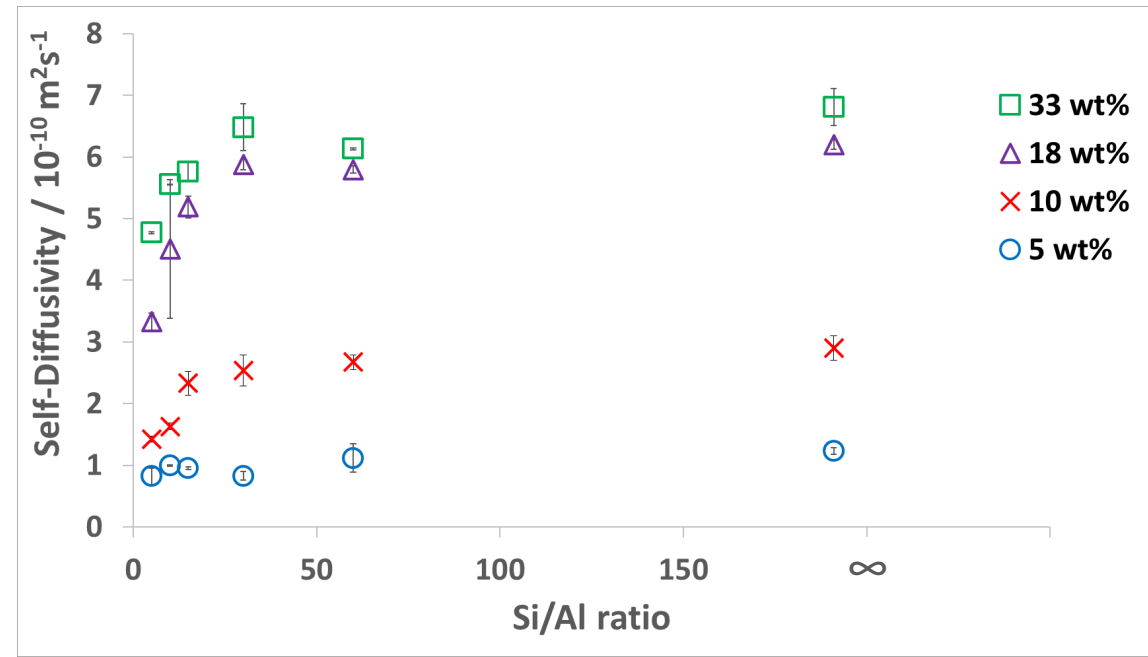

Figure S6. Diffusion coefficient of water at 5, 10, 18 and 33 wt\% loading within the silanol containing systems in the $\mathrm{Si} / \mathrm{Al}=191,60,30,15,10$ and 5 zeolite $\mathrm{Y}$ systems. 


\section{References}

1. Joshi, K. L.; Psofogiannakis, G.; van Duin, A. C. T.; Raman, S., Reactive molecular simulations of protonation of water clusters and depletion of acidity in H-ZSM-5 zeolite. Physical Chemistry Chemical Physics 2014, 16 (34), 18433-18441.

2. Krossner, M.; Sauer, J., Interaction of Water with Brønsted Acidic Sites of Zeolite Catalysts. Ab Initio Study of 1:1 and 2:1 Surface Complexes. The Journal of Physical Chemistry 1996, 100 (15), 6199-6211.

3. Farrell, J.; Manspeaker, C.; Luo, J., Understanding competitive adsorption of water and trichloroethylene in a high-silica Y zeolite. Microporous and Mesoporous Materials 2003, 59 (2), 205214.

4. Eckstein, S.; Hintermeier, P. H.; Zhao, R.; Baráth, E.; Shi, H.; Liu, Y.; Lercher, J. A., Influence of Hydronium lons in Zeolites on Sorption. Angewandte Chemie International Edition 2019, 58 (11), 3450-3455.

5. Mei, D.; Lercher, J. A., Mechanistic insights into aqueous phase propanol dehydration in $\mathrm{H}-$ ZSM-5 zeolite. AIChE Journal 2017, 63 (1), 172-184.

6. Van Duin, A. C.; Dasgupta, S.; Lorant, F.; Goddard, W. A., ReaxFF: a reactive force field for hydrocarbons. The Journal of Physical Chemistry A 2001, 105 (41), 9396-9409.

7. Bussai, C.; Vasenkov, S.; Liu, H.; Böhlmann, W.; Fritzsche, S.; Hannongbua, S.; Haberlandt, R.; Kärger, J., On the diffusion of water in silicalite-1: MD simulations using ab initio fitted potential and PFG NMR measurements. Applied Catalysis A: General 2002, 232 (1), 59-66.

8. Özgür Yazaydın, A.; Thompson, R. W., Molecular simulation of water adsorption in silicalite: Effect of silanol groups and different cations. Microporous and Mesoporous Materials 2009, 123 (1), 169-176.

9. $\quad$ Flanigen, E. M.; Bennett, J. M.; Grose, R. W.; Cohen, J. P.; Patton, R. L.; Kirchner, R. M.; Smith, J. V., Silicalite, a new hydrophobic crystalline silica molecular sieve. Nature 1978, 271 (5645), 512-516.

10. Giaya, A.; Thompson, R. W., Single-component gas phase adsorption and desorption studies using a tapered element oscillating microbalance. Microporous and Mesoporous Materials 2002, 55 (3), 265-274.

11. Olson, D. H.; Haag, W. O.; Borghard, W. S., Use of water as a probe of zeolitic properties: interaction of water with HZSM-5. Microporous and Mesoporous Materials 2000, 35-36, 435-446.

12. Ison, A.; Gorte, R. J., The adsorption of methanol and water on H-ZSM-5. Journal of Catalysis 1984, 89 (1), 150-158.

13. Vigne-Maeder, F.; Auroux, A., Potential maps of methane, water, and methanol in silicalite. The Journal of Physical Chemistry 1990, 94 (1), 316-322.

14. Ahunbay, M. G., Monte Carlo Simulation of Water Adsorption in Hydrophobic MFI Zeolites with Hydrophilic Sites. Langmuir 2011, 27 (8), 4986-4993. 